\title{
Ionothermal synthesis, structures and magnetism of three new open framework iron halide-phosphates
}

Guangmei Wang, ${ }^{a}$ Martin Valldor, ${ }^{b}$ Stefanie Siebeneichler, ${ }^{c}$ Magdalena Wilk-Kozubek,,${ }^{c, d}$ Volodymyr Smetana, ${ }^{c}$ and Anja-Verena Mudring ${ }^{*}, a, c$

${ }^{a}$ Fakultät für Chemie und Biochemie, Ruhr-Universität Bochum, Universitätsstr. 150, D-44780 Bochum.

${ }^{b}$ Leibniz-Institute for Solid State and Materials Research Dresden, Helmholtzstraße 20, 01069 Dresden, Germany.

c Department of Materials and Environmental Chemistry, Stockholm University, Svante Arrhenius väg 16 C, 10691 Stockholm, Sweden.

${ }^{d}$ ŁUKASIEWICZ Research Network - PORT Polish Center for Technology Development, 147 Stabłowicka Street, 54-066 Wrocław, Poland.

\section{Supplementary Information}



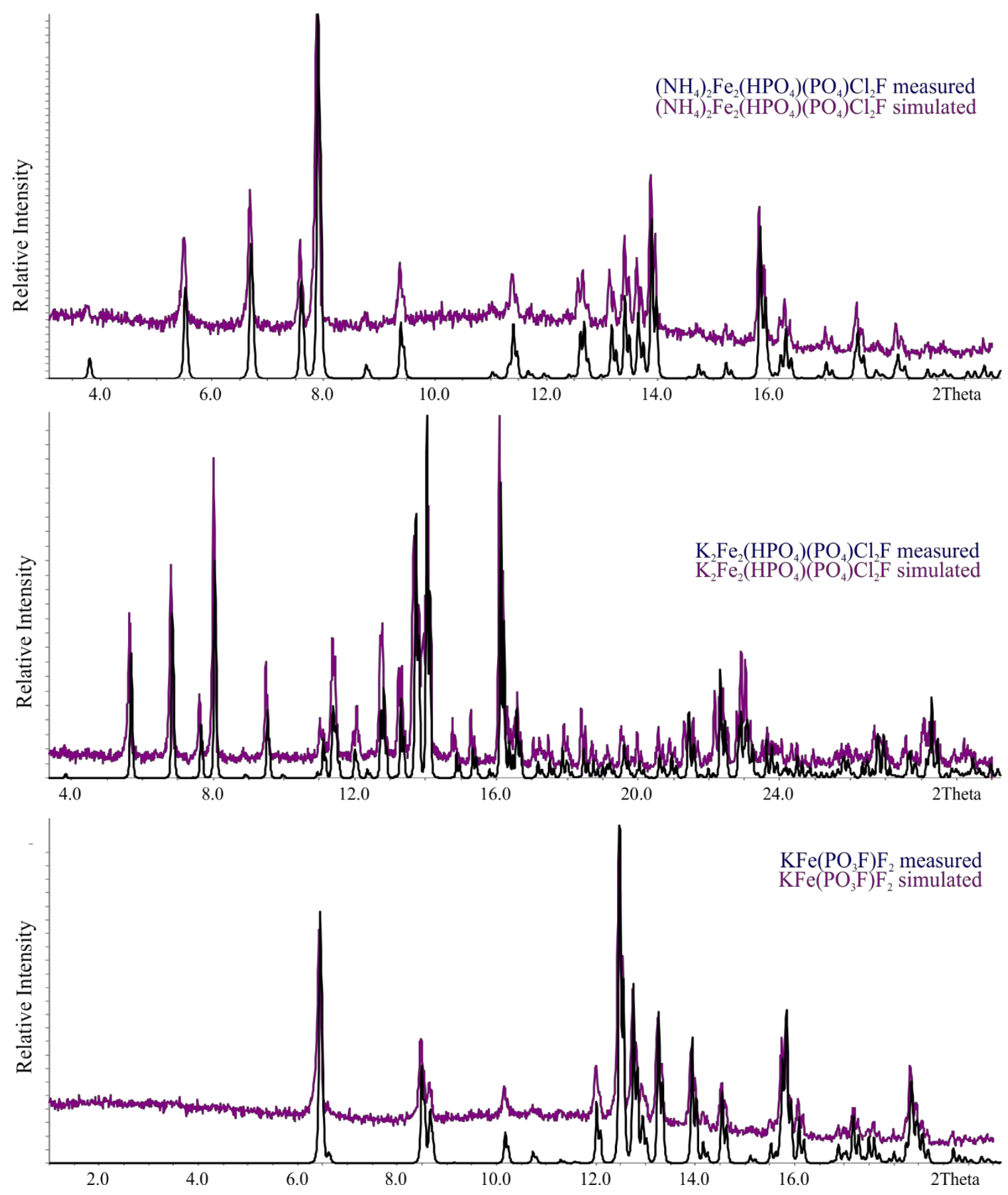

Figure S1. PXRD patterns of 1-3. 


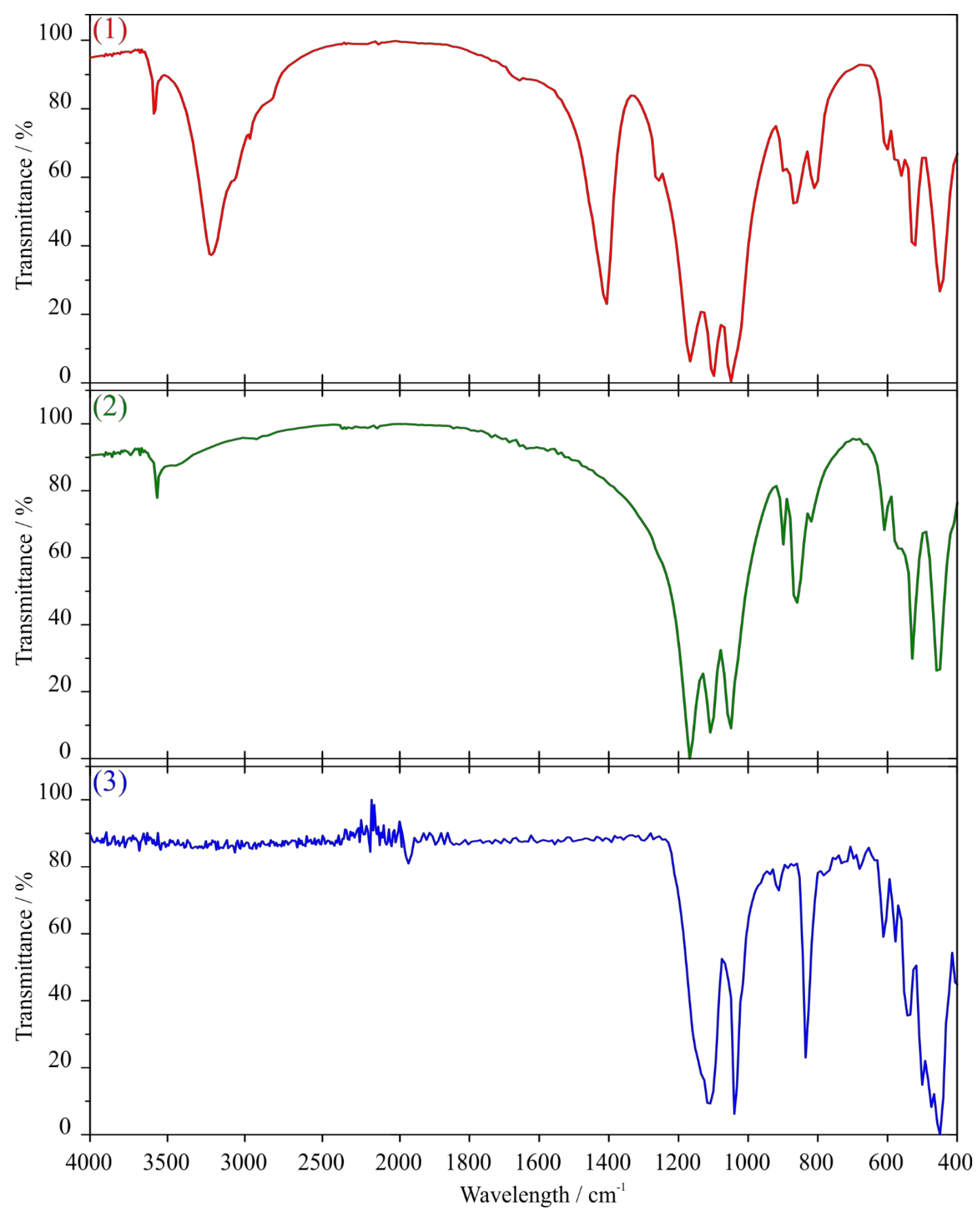

Figure S2. FTIR transmittance spectra of 1-3. 


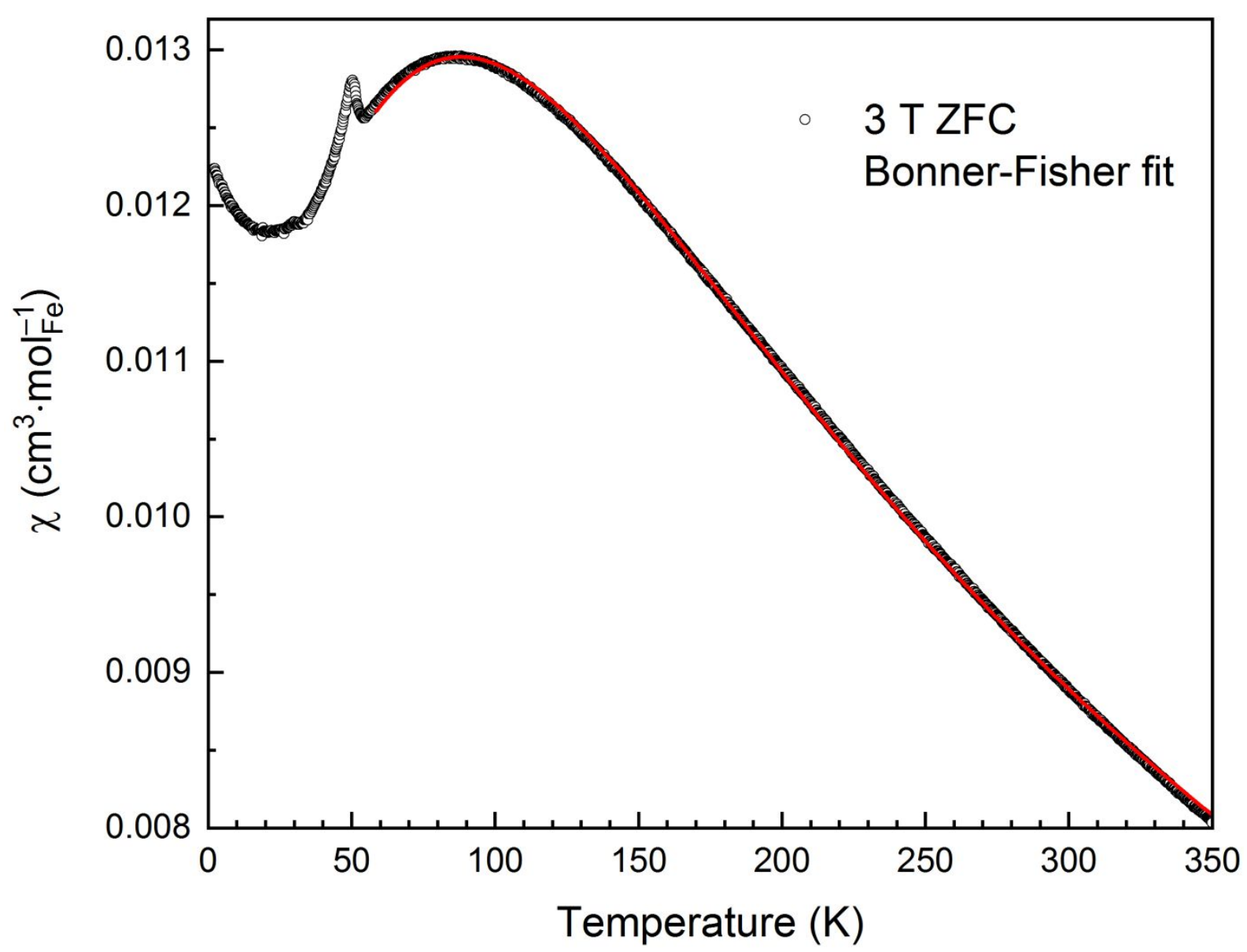

Figure S3. Bonner-Fisher fitting $(g=2)$ of the magnetic susceptibility data of $\mathbf{3}$. An additional scaling factor was added to the formula to achieve a good fit to the data $\left(J / k_{B}=8.02\right)$. The exact physical meaning of this factor is not clear since it could originate from impurities in the sample, a divergence from the theoretical model or the g-factor significantly deviating from 2. Electron spin resonance (ESR) measurements would be necessary to determine the actual value of $g$. 
Table S1. Atomic positions and equivalent displacement parameters for $\left(\mathrm{NH}_{4}\right)_{2} \mathrm{Fe}_{2}\left(\mathrm{HPO}_{4}\right)\left(\mathrm{PO}_{4}\right) \mathrm{Cl}_{2} \mathrm{~F}(\mathbf{1}), \mathrm{K}_{2} \mathrm{Fe}_{2}\left(\mathrm{HPO}_{4}\right)\left(\mathrm{PO}_{4}\right) \mathrm{Cl}_{2} \mathrm{~F}(\mathbf{2})$ and $\mathrm{KFe}\left(\mathrm{PO}_{3} \mathrm{~F}\right) \mathrm{F}_{2}(\mathbf{3})$

\begin{tabular}{|c|c|c|c|c|c|}
\hline \multicolumn{6}{|c|}{ Atomic parameters } \\
\hline Atom & Wyckoff site & $x$ & $y$ & $z$ & $U_{\mathrm{eq}}$ \\
\hline \multicolumn{6}{|c|}{$\left(\mathrm{NH}_{4}\right)_{2} \mathrm{Fe}_{2}\left(\mathrm{HPO}_{4}\right)\left(\mathrm{PO}_{4}\right) \mathrm{Cl}_{2} \mathrm{~F}$} \\
\hline N1 & $2 c$ & 0.75 & $0.264(1)$ & $0.1753(9)$ & $0.022(2)$ \\
\hline N2 & $2 c$ & 0.75 & $0.211(1)$ & $0.773(1)$ & $0.026(2)$ \\
\hline $\mathrm{Fe} 1$ & $2 c$ & 0.75 & $0.7432(1)$ & $0.9748(2)$ & $0.0111(3)$ \\
\hline $\mathrm{Fe} 2$ & $2 a$ & 0 & 0 & $0.46381(9)$ & $0.0075(3)$ \\
\hline $\mathrm{P} 1$ & $2 c$ & 0.75 & $0.7356(3)$ & $0.2910(2)$ & $0.0089(6)$ \\
\hline $\mathrm{P} 2$ & $2 c$ & 0.75 & $0.7612(2)$ & $0.6574(2)$ & $0.0096(5)$ \\
\hline H1 & $2 c$ & 0.75 & $0.479(6)$ & $0.569(4)$ & 0.05 \\
\hline $\mathrm{O} 1$ & $2 c$ & 0.75 & $0.6822(9)$ & $0.1571(6)$ & $0.019(1)$ \\
\hline $\mathrm{O} 2$ & $2 c$ & 0.75 & $0.5520(8)$ & $0.6421(7)$ & $0.033(1)$ \\
\hline $\mathrm{O} 3$ & $2 c$ & 0.75 & $0.7962(10)$ & $0.7935(6)$ & $0.027(2)$ \\
\hline $\mathrm{O} 4$ & $2 c$ & 0.75 & $0.5506(8)$ & $0.3610(7)$ & $0.033(1)$ \\
\hline O5 & $4 d$ & $0.9238(6)$ & $0.8310(7)$ & $0.3321(6)$ & $0.029(1)$ \\
\hline O6 & $4 d$ & $0.9238(8)$ & $0.8266(7)$ & $0.5948(6)$ & $0.033(1)$ \\
\hline $\mathrm{C} 11$ & $2 a$ & 0 & 0 & $0.0000(2)$ & $0.0177(5)$ \\
\hline $\mathrm{Cl} 2$ & $2 b$ & 0 & 0.5 & $0.9431(2)$ & $0.0192(5)$ \\
\hline F1 & $2 c$ & 0.75 & $0.1074(5)$ & $0.4656(6)$ & $0.0139(8)$ \\
\hline \multicolumn{6}{|c|}{$\mathrm{K}_{2} \mathrm{Fe}_{2}\left(\mathrm{HPO}_{4}\right)\left(\mathrm{PO}_{4}\right) \mathrm{Cl}_{2} \mathrm{~F}$} \\
\hline $\mathrm{K} 1$ & $4 c$ & $0.73253(16)$ & $0.62201(9)$ & $0.74729(14)$ & $0.0279(3)$ \\
\hline $\mathrm{K} 2$ & $4 c$ & $0.78621(17)$ & $0.64594(8)$ & $0.14837(13)$ & $0.0269(3)$ \\
\hline $\mathrm{Fe} 1$ & $4 c$ & $0.75337(7)$ & $0.87906(4)$ & $0.94826(8)$ & $0.00944(15)$ \\
\hline $\mathrm{Fe} 2$ & $4 c$ & $0.51059(7)$ & $0.74932(4)$ & $0.45994(6)$ & $0.00709(14)$ \\
\hline $\mathrm{P} 1$ & $4 c$ & $0.74824(13)$ & $0.88678(9)$ & $0.63147(10)$ & $0.0080(3)$ \\
\hline $\mathrm{P} 2$ & $4 c$ & $0.76538(14)$ & $0.87230(7)$ & $0.26735(11)$ & $0.0080(2)$ \\
\hline H1 & $4 c$ & $0.661(5)$ & $0.990(3)$ & $0.4874(15)$ & 0.03 \\
\hline $\mathrm{O} 1$ & $4 c$ & $0.8015(5)$ & $0.9809(2)$ & $0.2883(4)$ & $0.0199(7)$ \\
\hline $\mathrm{O} 2$ & $4 c$ & $0.7787(4)$ & $0.9125(2)$ & $0.7666(3)$ & $0.0187(7)$ \\
\hline $\mathrm{O} 3$ & $4 c$ & $0.5828(4)$ & $0.8227(2)$ & $0.6114(3)$ & $0.0148(6)$ \\
\hline $\mathrm{O} 4$ & $4 c$ & $0.9250(4)$ & $0.8514(2)$ & $0.5692(3)$ & $0.0173(7)$ \\
\hline O5 & $4 c$ & $0.7026(5)$ & $0.9832(2)$ & $0.5657(3)$ & $0.0175(6)$ \\
\hline O6 & $4 c$ & $0.6000(4)$ & $0.8511(2)$ & $0.3518(3)$ & $0.0145(6)$ \\
\hline O7 & $4 c$ & $0.9437(4)$ & $0.8221(2)$ & $0.3049(3)$ & $0.0196(7)$ \\
\hline O8 & $4 c$ & $0.7206(5)$ & $0.8553(3)$ & $0.1321(3)$ & $0.0202(7)$ \\
\hline C11 & $2 a$ & 0 & 0 & $0.99998(19)$ & $0.0207(3)$ \\
\hline $\mathrm{C} 12$ & $2 b$ & 0 & 0.5 & $0.9633(2)$ & $0.0272(4)$ \\
\hline $\mathrm{Cl} 3$ & $4 c$ & $0.99822(14)$ & $0.75001(7)$ & $0.92259(13)$ & $0.0189(3)$ \\
\hline $\mathrm{F}$ & $4 c$ & $0.7612(3)$ & $0.69160(16)$ & $0.4593(3)$ & $0.0133(5)$ \\
\hline \multicolumn{6}{|c|}{$\mathrm{KFe}\left(\mathrm{PO}_{3} \mathrm{~F}\right) \mathrm{F}_{2}$} \\
\hline K1 & $4 e$ & $0.6393(3)$ & $0.30247(8)$ & $0.18447(16)$ & $0.0209(3)$ \\
\hline $\mathrm{Fe} 1$ & $2 b$ & 0.5 & 0 & 0 & $0.0053(2)$ \\
\hline $\mathrm{Fe} 2$ & $2 c$ & 0 & 0 & 0.5 & $0.0069(2)$ \\
\hline $\mathrm{P} 1$ & $4 e$ & $0.1113(2)$ & $0.10731(9)$ & $0.17968(15)$ & $0.0069(3)$ \\
\hline $\mathrm{F} 1$ & $4 e$ & $0.3213(5)$ & $0.44822(19)$ & $0.2383(3)$ & $0.0101(6)$ \\
\hline F2 & $4 e$ & $0.1956(6)$ & $0.2196(2)$ & $0.2743(4)$ & $0.0186(6)$ \\
\hline F3 & $4 e$ & $0.1515(6)$ & $0.6379(2)$ & $0.0454(4)$ & $0.0163(6)$ \\
\hline $\mathrm{O} 1$ & $4 e$ & $0.2079(6)$ & $0.6132(2)$ & $0.4437(4)$ & $0.0090(6)$ \\
\hline $\mathrm{O} 2$ & $4 e$ & $0.2692(6)$ & $0.0995(2)$ & $0.0631(4)$ & $0.0110(6)$ \\
\hline $\mathrm{O} 3$ & $4 e$ & $0.2018(6)$ & $0.0306(3)$ & $0.3432(4)$ & $0.0119(7)$ \\
\hline
\end{tabular}

